TRANSACTIONS OF THE

AMERICAN MATHEMATICAL SOCIETY

Volume 351, Number 10, Pages 4183-4203

S 0002-9947(99)02384-3

Article electronically published on April 20, 1999

\title{
MINIMAL LATTICE-SUBSPACES
}

\author{
IOANNIS A. POLYRAKIS
}

\begin{abstract}
In this paper the existence of minimal lattice-subspaces of a vector lattice $E$ containing a subset $B$ of $E_{+}$is studied (a lattice-subspace of $E$ is a subspace of $E$ which is a vector lattice in the induced ordering). It is proved that if there exists a Lebesgue linear topology $\tau$ on $E$ and $E_{+}$is $\tau$-closed (especially if $E$ is a Banach lattice with order continuous norm), then minimal lattice-subspaces with $\tau$-closed positive cone exist (Theorem 2.5).

In the sequel it is supposed that $B=\left\{x_{1}, x_{2}, \ldots, x_{n}\right\}$ is a finite subset of $C_{+}(\Omega)$, where $\Omega$ is a compact, Hausdorff topological space, the functions $x_{i}$ are linearly independent and the existence of finite-dimensional minimal lattice-subspaces is studied. To this end we define the function $\beta(t)=\frac{r(t)}{\|r(t)\|_{1}}$ where $r(t)=\left(x_{1}(t), x_{2}(t), \ldots, x_{n}(t)\right)$. If $R(\beta)$ is the range of $\beta$ and $K$ the convex hull of the closure of $R(\beta)$, it is proved:

(i) There exists an $m$-dimensional minimal lattice-subspace containing $B$ if and only if $K$ is a polytope of $\mathbb{R}^{n}$ with $m$ vertices (Theorem 3.20).

(ii) The sublattice generated by $B$ is an $m$-dimensional subspace if and only if the set $R(\beta)$ contains exactly $m$ points (Theorem 3.7).

This study defines an algorithm which determines whether a finite-dimensional minimal lattice-subspace (sublattice) exists and also determines these subspaces.
\end{abstract}

\section{INTRODUCTION}

It is known that $C[0,1]$ is a universal Banach space in the sense that every separable Banach space is isometric to a closed subspace of $C[0,1]$. In [11] it is shown that each separable Banach lattice is order-isomorphic to a closed latticesubspace of $C[0,1]$; therefore $C[0,1]$ is also a universal Banach lattice. Since the sublattices of $C[0,1]$ are not enough for this representation, the lattice-subspaces seems to be the right class of subspaces for studying Banach lattices.

The structure of lattice-subspaces has not been systematically studied. In [7] it is shown that a subspace $X$ of a vector lattice is a lattice-subspace if and only if there exists a positive projection from the vector sublattice generated by $X$ onto $X$. In [10] and [11] the existence of positive bases in lattice-subspaces is studied. A survey of lattice-subspaces and positive projections, as well as some new results, is proved in [1]. In [12] the finite-dimensional lattice-subspaces of $C(\Omega)$ are studied.

In the present paper the existence of minimal lattice-subspaces of a vector lattice $E$ which contains a subset $B$ of $E_{+}$is studied. In the theory of Banach lattices (and

Received by the editors March 16, 1997.

1991 Mathematics Subject Classification. Primary 46B42, 52A21, 15A48, 53A04.

This research was supported by the 1995 PENED program of the Ministry of Industry, Energy and Technology of Greece. 
in applications) we are interested in a lattice-subspace of $E$ containing $B$ which is as "close" as possible to the linear subspace $[B]$ generated by $B$.

Such a subspace is the sublattice $S(B)$ generated by $B$ (note that $S(B)$ is the minimum sublattice containing $B$ and also that $S(B)=[B]^{\vee}-[B]^{\vee}$ where $[B]^{\vee}$ is the set of finite supremum of the elements of $[B]$ ) but $S(B)$ is in general a "big" subspace which is "very far" from $[B]$. In Example $3.18[B]$ is 3-dimensional, $S(B)$ is dense in $C(\Omega)$ but a 4 -dimensional lattice-subspace containing $B$ exists. In Example 3.21 it is shown that a minimum lattice-subspace containing $B$ does not always exist.

An important question is "how far" a minimal lattice-subspace is from $[B]$. Motivated by this question we study the existence of finite-dimensional minimal lattice-subspaces. Especially we suppose that $B=\left\{x_{1}, x_{2}, \ldots, x_{n}\right\}$ is a subset of $C_{+}(\Omega)$, the vectors $x_{i}$ are linearly independent and we study the existence of finitedimensional minimal lattice-subspaces of $C(\Omega)$ containing $B$. In the framework of this problem we study also the question whether $S(B)$ is a finite-dimensional subspace.

To study this problem we define the function $\beta(t)=\frac{r(t)}{\|r(t)\|_{1}}$ where $r(t)=$ $\left(x_{1}(t), x_{2}(t), \ldots, x_{n}(t)\right)$. This function defines a curve in the simplex $\Delta_{n}$ of $\mathbb{R}_{+}^{n}$ which we call basic curve of the functions $x_{i}$ and is very important for our study.

In Theorem 3.7 it is proved that $S(B)$ is finite-dimensional if and only if the range $R(\beta)$ of $\beta$ is finite and a positive basis of $S(B)$ is also determined. Hence we can determine whether $S(B)$ is finite-dimensional because it is very easy to check if $R(\beta)$ is finite or not. By the property that $S(B)=[B]^{\vee}-[B]^{\vee}$ we cannot conclude whether $S(B)$ is finite-dimensional and also we cannot determine a positive basis of $S(B)$.

In Theorem 3.10 it is proved that if the convex hull $K$ of the closure of $R(\beta)$ is a polytope with $m$ vertices, then an $m$-dimensional minimal lattice-subspace $Y$ exists and a positive basis of $Y$ is given. The determination of the basis of $Y$ is based on the determination of the vertices of $K$.

In general it is difficult to study whether $K$ is a polytope or not and determine its vertices. In Corollary 3.15 it is proved that if $K$ is a polytope, $\beta\left(t_{0}\right)$ a vertex of $K$ and $t_{0}$ an interior point of a curve $c$ of $\Omega$, then the derivative at $t_{0}$ (whenever it exists) of the restriction of $\beta$ on $c$ is equal to zero. If for example $\Omega \subseteq \mathbb{R}^{l}$ and the function $\beta$ is defined on the whole set $\Omega$, then the partial derivatives of $\beta$ at $t_{0}$ are equal to zero whenever $t_{0}$ is an interior point of $\Omega$ and the derivatives at $t_{0}$ of the restriction of $\beta$ on the parametric curves of $\partial(\Omega)$ are equal to zero, if $t_{0} \in \partial(\Omega)$. Hence $t_{0}$ can be obtained as a solution of a system of equations.

This property helps us to determine a set of possible vertices of $K$, i.e., a subset $G$ of $\mathbb{R}^{n}$ which contains the vertices of $K$, whenever $K$ is a polytope. After the determination of $G$ it is easier to study if $K$ is a polytope or not (see Algorithm 3.17 and Example 3.18).

An interesting remark on the structure of the lattice-subspaces is also that a minimal lattice-subspace containing $B$ is not necessarily a subspace of $S(B)$, Example 3.21 .

Recently lattice-subspaces have been employed in economics [2], [3].

Let $E$ be a (partially) ordered vector space with positive cone $E_{+}$and $X$ a subspace of $E$. The cone $X \cap E_{+}$will be called the induced cone of $X$, and the ordering defined in $X$ by this cone the induced ordering. We will denote by $X_{+}$the 
induced cone of $X$, i.e., $X_{+}=X \cap E_{+}$. An ordered subspace of $E$ is a subspace of $E$ ordered by the induced cone. A lattice-subspace of $E$ is an ordered subspace of $E$ which is also a vector lattice (Riesz space).

Let $X$ be a lattice-subspace of $E$. Then, for each $x, y \in X$ we will denote by $x \nabla y$ (resp. $x \triangle y$ ) the supremum (resp. infimum) of $\{x, y\}$ in $X$. It is clear that

$$
x \vee y \leq x \nabla y \text { and } x \triangle y \leq x \wedge y
$$

whenever $x \vee y, x \wedge y$ exist. If $E$ is a vector lattice and $x \nabla y=x \vee y$ for any $x, y \in X$ then $X$ is a sublattice (Riesz subspace) of $E$. Let $E$ be an ordered Banach space with positive cone $E_{+}$. A sequence $\left\{e_{n}\right\}$ is a positive basis of $E$ if $\left\{e_{n}\right\}$ is a (Schauder) basis of $E$ and $E_{+}=\left\{x=\sum_{i=1}^{\infty} \lambda_{i} e_{i} \mid \lambda_{i} \in \mathbb{R}_{+}\right.$for each $\left.i\right\}$. A positive basis $\left\{e_{n}\right\}$ of $E$ is unique (in the sense of a positive multiple). The following result (see [1] or [12]) is very important for the study of finite-dimensional lattice-subspaces. It can be proved either elementary or as a partial result of the Choquet-Kentall Theorem.

Theorem 1.1. A finite-dimensional ordered vector space $E$ is a vector lattice if and only if $E$ has a positive basis.

For notation and terminology not defined here we refer to $[4,6,9]$.

\section{Minimal lattice-SubSPaces}

Let $E$ be a vector lattice and $B \subseteq E_{+}, B \neq \emptyset$. Let $L$ be the set of latticesubspaces of $E$, each of which contains $B$. If $X \in L$ and for any $Y \in L$ it holds:

$$
Y \subseteq X \Rightarrow Y=X,
$$

then we will say that $X$ is a minimal lattice-subspace of $E$ containing $B$.

If $E$ is a vector lattice, then the sublattice generated by $B$ is the minimum sublattice containing $B$.

As we will show later (Example 3.21) even if $E=\mathbb{R}^{m}$ a minimum lattice-subspace of $E$ containing $B$ does not always exist. So we state the following question:

Problem 2.1. Does a minimal lattice subspace of $E$ containing $B$ exist?

Let $P$ be a cone of a linear space $F$ (i.e., $P$ is a convex subset of $F, \lambda x \in P$ for each $x \in P$ and $\lambda \in \mathbb{R}_{+}$and $\left.P \cap(-P)=\{0\}\right)$. Suppose that $x, y \in P$. If there exists $z \in P$ with the properties: $z-x, z-y \in P$ and for each $w \in P, w-x, w-y \in P$ imply that $w-z \in P$, then we will say that $z$ is the supremum of $\{x, y\}$ in $P$ and we will denote

$$
z=\sup _{P}\{x, y\} \text {. }
$$

The infimum of $\{x, y\}$ in $P$ is defined analogously. If for each $x, y \in P, z=$ $\sup _{P}\{x, y\}$ exists, then $\inf _{P}\{x, y\}$ also exists.

If $P$ is a cone of a linear space $F$ and for each $x, y \in P$ the supremum of $\{x, y\}$ exists in $P$, then we will say that $P$ is a lattice cone of $F$.

If $x=x_{1}-x_{2}$ where $x_{1}, x_{2} \in P$, then it is easy to show that $\sup \{x, 0\}=$ $\sup _{P}\left\{x_{1}, x_{2}\right\}-x_{2}$ is the supremum of $\left\{x_{1}, x_{2}\right\}$ in $X=P-P$. Therefore the following result holds.

$A$ cone $P$ of a vector space $F$ is a lattice-cone if and only if the subspace $X=$ $P-P$, ordered by the cone $P$, is a vector lattice.

In the next results of this paragraph we will suppose that $E$ is a vector lattice equipped with a linear topology $\tau$ with the properties: 
(i) $E_{+}$is $\tau$-closed;

(ii) each increasing, order bounded net of $E$ has a $\tau$-convergent subnet (i.e., the topology $\tau$ is Lebesgue).

Property (i) implies also that $\tau$ is Hausdorff because if we suppose that $x \in E$, $x \neq 0$ and $0 \in x+V$ for each open symmetric neighborhood $V$ of zero, then $0 \in-x+V$; therefore $x$ and $-x$ belong to $E_{+}$and hence $x=0$, contradiction.

If the topology $\tau$ is order continuous (i.e., each decreasing net of $E$ with infimum zero is $\tau$-convergent to zero) and $E$ is Dedekind complete, then $\tau$ satisfies (ii). If the order intervals of $E$ are $\tau$-compact, the statement (ii) is also satisfied (for related results see [4, Theorem 11.13]). Hence, the weak star topology of a dual Banach lattice and the weak topology of a Banach lattice with order continuous norm [4, Theorem 12.9], have property (ii).

Proposition 2.2. Let $\left(P_{i}\right)_{i \in I}$ be a decreasing net of $\tau$-closed lattice cones of $E_{+}$ (i.e., $P_{i} \subseteq E_{+}$and $i \preceq j \Rightarrow P_{i} \supseteq P_{j}$ ). Then $P=\bigcap_{i \in I} P_{i}$ is a $\tau$-closed lattice cone of $E$.

Proof. $P$ is a $\tau$-closed cone of $E_{+}$. Let $x, y \in P$. Denote by $z_{i}$ the supremum of $\{x, y\}$ in $P_{i}$. For each $i, j \in I$ with $i \preceq j$ we have $P_{j} \subseteq P_{i} \subseteq E_{+}$; therefore,

$$
x, y \leq z_{i} \leq z_{j} \leq x+y .
$$

Since $\tau$ has property (ii), there exists a $\tau$-convergent subnet of $\left(z_{i}\right)_{i \in I}$ which we will still denote by $\left(z_{i}\right)_{i \in I}$. This net is also increasing, and let $z=\lim _{i \in I} z_{i}$. Let $i \in I$. Then for each $j \in I$ with $i \preceq j$, we have:

$$
z_{j}, z_{j}-x, z_{j}-y \in P_{j} \subseteq P_{i}
$$

Since the cone $P_{i}$ is $\tau$-closed, we have that

$$
z, z-x, z-y \in P_{i}, \quad \text { for each } i \in I \text {. }
$$

Therefore

$$
z, z-x, z-y \in P \text {. }
$$

Suppose that $w \in P$ with $w-x, w-y \in P$. Since $P \subseteq P_{j}$ we have that $w-z_{j} \in$ $P_{j} \subseteq P_{i}$ for each $j \in I$ with $i \preceq j$. Hence $w-z \in P_{i}$ for each $i$; therefore $w-z \in P$. So we have proved that $z=\sup _{P}\{x, y\}$; therefore $P$ is a lattice cone.

Theorem 2.3. Let $P \subseteq E_{+}$be a cone and let $\Phi(P)$ be the set of $\tau$-closed lattice cones of $E_{+}$each of which contains $P$. Then $\Phi(P)$ has minimal elements.

Proof. $\Phi(P) \neq \emptyset$ because $E_{+} \in \Phi(P)$ and $\Phi(P)$, ordered by the relation " $\supseteq$ ", is a partially ordered set. Suppose that $\mathcal{F}$ is a totally ordered subset of $\Phi(P)$. Then by the previous result $Q=\bigcap_{A \in \mathcal{F}} A$ is a $\tau$-closed lattice cone of $E$. By Zorn's Lemma the theorem is true.

Proposition 2.4. Let $\left(X_{i}\right)_{i \in I}$ be a decreasing net of lattice-subspaces of $E$ with $\tau$-closed positive cones. Let $X=\bigcap_{i \in I} X_{i}, Y=X_{+}-X_{+}$and $Y_{+}=Y \cap E_{+}$. Then

(i) $X_{+}=\bigcap_{i \in I} X_{i}^{+}$.

(ii) $Y \subseteq X, Y_{+}=X_{+}$and $Y$ is a lattice-subspace of $E$ with $\tau$-closed positive cone. 
Proof. (i) $X_{+}=X \cap E_{+}=\left(\bigcap_{i \in I} X_{i}\right) \cap E_{+}=\bigcap_{i \in I} X_{i}^{+}$.

(ii) $Y=X_{+}-X_{+} \subseteq X$. $Y_{+} \subseteq X \cap E_{+}=X_{+}$. Also $X_{+}=X_{+}-\{0\} \subseteq Y$; therefore $X_{+} \subseteq Y_{+}$. Hence $X_{+}=Y_{+}$. The net $\left(X_{i}^{+}\right)_{i \in I}$ is a decreasing net of $\tau$-closed lattice cones of $E_{+}$; therefore $Y_{+}$is a $\tau$-closed lattice cone. Hence $Y$, is a lattice-subspace of $E$.

Theorem 2.5. Let $B \subseteq E_{+}$and

$$
l(B)=\left\{Y \subseteq E \mid Y \text { is a lattice-subspace, } Y_{+} \text {is } \tau \text {-closed and } B \subseteq Y\right\} .
$$

Then $l(B)$ has minimal elements.

Proof. The set $l(B)$ is nonempty because it contains $E$. The set $l(B)$, ordered by the relation " $\supseteq$ ", is a partially ordered set. Let $\mathcal{F}$ be a totally ordered subset of $l(B)$. By the previous proposition there exists $Y \in l(B)$ such that $Y \subseteq A$ for each $A \in \mathcal{F}$. Therefore, by Zorn's Lemma $l(B)$ has minimal elements.

Corollary 2.6. Let $E$ be a Banach lattice with order continuous norm and $B \subseteq$ $E_{+}$. Then the set of lattice-subspaces of $E$ with (norm) closed positive cone which contains $B$ has minimal elements.

\section{The Finite-Dimensional CASE IN $C(\Omega)$}

In this paper we shall denote by $\Omega$ a compact, Hausdorff topological space and by $C(\Omega)$ the Banach lattice of continuous real valued functions defined on $\Omega$.

We will also denote by $x_{1}, \ldots, x_{n}, n$ fixed linearly independent positive elements of $C(\Omega)$ and by $X$ the subspace of $C(\Omega)$ generated by $x_{1}, \ldots, x_{n}$, i.e.,

$$
X=\left[x_{1}, x_{2}, \ldots, x_{n}\right] .
$$

In [12] necessary and sufficient conditions in order for $X$ to be a lattice-subspace of $C(\Omega)$ are given.

In this paper we study the problem:

Problem 3.1. Does a finite-dimensional lattice-subspace (sublattice) of $C(\Omega)$ containing $x_{1}, x_{2}, \ldots, x_{n}$ exist?

For each $x \in \mathbb{R}^{m}$ we will denote by $x(i)$ the $i$-coordinate of $x$, by $\|x\|_{1}$ the norm $\|x\|_{1}=\sum_{i=1}^{m}|x(i)|$, by $\left\{e_{1}, e_{2}, \ldots, e_{m}\right\}$ the usual basis of $\mathbb{R}^{m}$ and by $\Delta_{m}$ the simplex (base) of $\mathbb{R}_{+}^{m}$, i.e.,

$$
\Delta_{m}=\left\{x \in \mathbb{R}_{+}^{m} \mid\|x\|_{1}=1\right\} .
$$

Also if $x \in \mathbb{R}^{m}, y \in \mathbb{R}^{l}$ we shall denote by $(x, y)$ the vector $z$ of $\mathbb{R}^{m+l}$ with $z(i)=x(i)$ for $i=1,2, \ldots, m$ and $z(m+i)=b(i)$ for $i=1,2, \ldots, l$. If $A$ is an $m \times m$ matrix we shall denote by $A^{T}$ the transpose and by $A^{-1}$ the inverse matrix of $A$.

Let $y_{1}, y_{2}, \ldots, y_{m} \in C_{+}(\Omega)$. Then we will call the function $v(t)=\left(y_{1}(t), y_{2}(t)\right.$, $\left.\ldots, y_{m}(t)\right), t \in \Omega$, the curve and the function $\gamma(t)=\frac{v(t)}{\|v(t)\|_{1}}, t \in \Omega$, with $v(t) \neq 0$, the basic curve of $y_{1}, y_{2}, \ldots, y_{m}$. We will denote by $D(\gamma)$ the domain and by $R(\gamma)$ the range of $\gamma$. It is clear that $D(\gamma)$ is an open subset of $\Omega$ and $R(\gamma) \subseteq \Delta_{m}$.

In this paper we will denote by $r$ the curve and by $\beta$ the basic curve of $x_{1}, x_{2}, \ldots$, $x_{n}$, i.e.,

$$
r(t)=\left(x_{1}(t), x_{2}(t), \ldots, x_{n}(t)\right), \quad t \in \Omega \quad \text { and } \beta(t)=\frac{r(t)}{\|r(t)\|_{1}} .
$$


As usual if $K$ is a subset of a topological space $F$, we shall denote by $\operatorname{int}(K)$ the interior, by $\bar{K}$ the closure and by $\partial(K)$ the boundary of $K$. Also whenever $F$ is a linear topological space we shall denote by co $K$ the convex hull of $K$, by $\overline{\operatorname{co}} K$ the closure of co $K$ and by ep $(K)$ the set of extreme points of $K$.

Proposition 3.2 ([12, Proposition 2.2]). Let $Y$ be a lattice-subspace of $C(\Omega)$ with a positive basis $\left\{b_{1}, b_{2}, \ldots, b_{n}\right\}$. Then $Y$ is a sublattice of $C(\Omega)$ if and only if the sets $b_{i}^{-1}(0,+\infty)=\left\{t \in \Omega \mid b_{i}(t)>0\right\}, i=1,2, \ldots, n$, are pairwise disjoint.

Theorem 3.3 ([12, Theorem 3.6]). The statements (i) and (ii) are equivalent:

(i) $X$ is a lattice-subspace of $C(\Omega)$.

(ii) There exist $n$ linearly independent vectors $P_{1}, P_{2}, \ldots, P_{n}$ of $\mathbb{R}^{n}$, belonging to the closure of the range of $\beta$ such that for each $t \in D(\beta)$ the vector $\beta(t)$ is a convex combination of the vectors $P_{1}, P_{2}, \ldots, P_{n}$.

If the statement (ii) is true, $A$ is the $n \times n$ matrix whose ith column is the vector $P_{i}$ and $b_{1}, b_{2}, \ldots, b_{n}$ are the functions defined by the formula

$$
\left(b_{1}, b_{2}, \ldots, b_{n}\right)^{T}=A^{-1}\left(x_{1}, x_{2}, \ldots, x_{n}\right)^{T},
$$

then $\left\{b_{1}, b_{2}, \ldots, b_{n}\right\}$ is a positive basis of $X$.

Lemma 3.4. The functions $y_{i} \in C_{+}(\Omega), i=1,2, \ldots, m$, are linearly independent if and only if the space generated by the range of the basic curve $\gamma$ of $y_{i}, i=$ $1,2, \ldots, m$, is $\mathbb{R}^{m}$.

Proof. Let $W$ be the subspace of $\mathbb{R}^{m}$ generated by $R(\gamma)$. Then $W$ is also generated by the range of the curve $v$ of $y_{i}, i=1,2, \ldots, m$. Let $\left\{u_{i}=v\left(t_{i}\right) \mid i=1,2, \ldots, l\right\}$ be a basis of $W$. Then $l \leq m$.

Suppose that the functions $y_{i}$ are linearly independent. Then

$$
v(t)=\sum_{i=1}^{l} \xi_{i}(t) u_{i}, \quad \text { for each } t \in \Omega ;
$$

therefore

$$
y_{j}(t)=\sum_{i=1}^{l} \xi_{i}(t) u_{i}(j), \quad j=1,2, \ldots, m,
$$

where $u_{i}(j)$ is the $j$-coordinate of $u_{i}$. For each $t$, the vector $\left(\xi_{1}(t), \xi_{2}(t), \ldots, \xi_{l}(t)\right)$ is the unique solution of the system (2); therefore the functions $\xi_{i}$ as linear combinations of the functions $y_{i}$ belong to $C(\Omega)$. By $(2)$ we have also that

$$
y_{i} \in L=\left[\xi_{1}, \xi_{2}, \ldots, \xi_{l}\right], \quad \text { for each } i
$$

therefore $m \leq \operatorname{dim} L \leq l$. Hence $m=l$ and $W=\mathbb{R}^{m}$.

To prove the converse, suppose that $l=m$ and

$$
\sum_{i=1}^{m} \lambda_{i} y_{i}=0
$$

Then

$$
\sum_{i=1}^{m} \lambda_{i} y_{i}\left(t_{j}\right)=0 \quad \text { for each } j=1,2, \ldots, m \text {. }
$$


Since the vectors $v\left(t_{i}\right), i=1,2, \ldots, m$, are linearly independent, the system has the unique solution $\lambda_{i}=0$ for each $i$; therefore the functions $y_{i}$ are linearly independent.

\section{Sublattices.}

Theorem 3.5. Let $R(\beta)=\left\{P_{1}, P_{2}, \ldots, P_{n}\right\}$. (By the previous lemma the vectors $P_{i}$ are linearly independent and by Theorem $3.3 X$ is a lattice-subspace.) Let $\left\{b_{1}, b_{2}, \ldots, b_{n}\right\}$ be the positive basis of $X$ defined by (1) and let $I_{i}=b_{i}^{-1}(0,+\infty)$, for each $i$.

Then the following statements hold:

(i) $X$ is a sublattice of $C(\Omega)$.

(ii) $I_{i}=\beta^{-1}\left(P_{i}\right)$ for each $i$ and $D(\beta)=\bigcup_{i=1}^{n} I_{i}$.

(iii) If $y_{i}, i=1,2, \ldots, m$, are linearly independent elements of $X_{+}$and $\gamma$ is the basic curve of $y_{i}, i=1,2, \ldots, m$, then there exists $\Phi \subseteq\{1,2, \ldots, n\}$ such that

(a) $D(\gamma)=\bigcup_{i \in \Phi} I_{i}$

(b) the function $\gamma$ is constant on $I_{i}$ for each $i \in \Phi$,

(c) $m \leq l \leq n$, where $l$ is the cardinal number of $R(\gamma)$.

Proof. Let $z=\sum_{i=1}^{n} x_{i}$ and $B_{i}=\beta^{-1}\left(P_{i}\right), i=1,2, \ldots, n$. Then the sets $B_{i}$ are pairwise disjoint and $D(\beta)=\bigcup_{i=1}^{n} B_{i}$. By (1) we have that

$$
\frac{1}{z(t)}\left(b_{1}(t), b_{2}(t), \ldots, b_{n}(t)\right)^{T}=A^{-1}(\beta(t))^{T} .
$$

Since $A^{-1} \cdot A=I$, the dot-product of the $j$-row of $A^{-1}$ and the vector $P_{i}$ is equal to 1 if $i=j$ and 0 whenever $i \neq j$; therefore

$$
A^{-1}(\beta(t))^{T}=\left(e_{i}\right)^{T} \quad \text { for each } t \in B_{i},
$$

where $\left\{e_{1}, e_{2}, \ldots, e_{n}\right\}$ is the usual basis of $\mathbb{R}^{n}$. Therefore

$$
\frac{1}{z(t)}\left(b_{1}(t), b_{2}(t), \ldots, b_{n}(t)\right)=e_{i} \quad \text { for each } t \in B_{i} .
$$

Hence for each $t \in B_{i}$ it holds:

$$
z(t)=b_{i}(t)>0 \quad \text { and } \quad b_{j}(t)=0 \quad \text { for each } j \neq i .
$$

So

$$
B_{i} \subseteq I_{i} \quad \text { and } \quad B_{i} \cap I_{j}=\emptyset \quad \text { for each } j \neq i .
$$

Suppose that $t \in I_{i} \backslash B_{i}$. Since $D(\beta)=\bigcup_{k=1}^{n} B_{k}, t \in B_{j}$ for exactly one $j \neq i$. Hence $I_{i} \cap B_{j} \neq \emptyset$, contradiction. Hence $B_{i}=I_{i}$ for each $i$, and by Theorem 3.2, $X$ is a sublattice. We have also shown the statement (ii).

The basic curve $\gamma$ is

$$
\gamma(t)=\frac{1}{y(t)}\left(y_{1}(t), y_{2}(t), \ldots, y_{m}(t)\right)
$$

where $y=\sum_{i=1}^{m} y_{i}$. Let

$$
y_{j}=\sum_{i=1}^{n} \mu_{j i} b_{i}, \quad j=1,2, \ldots, m .
$$


Then $y=\sum_{i=1}^{n} \mu_{i} b_{i}$ where $\mu_{i}=\sum_{j=1}^{m} \mu_{j i}$ for each $i$. Let $\Phi=\left\{i \mid \mu_{i}>0\right\}$. Then it is clear that

$$
D(\gamma)=\bigcup_{i \in \Phi} I_{i}
$$

If $i \in \Phi$ and $t \in I_{i}$, then

$$
\gamma(t)=\frac{1}{\mu_{i}}\left(\mu_{1 i}, \mu_{2 i}, \ldots, \mu_{m i}\right)=Q_{i} ;
$$

hence $\gamma$ is constant on $I_{i}$. Therefore

$$
R(\gamma)=\left\{Q_{i} \mid i \in \Phi\right\}
$$

Since $\Phi$ is a subset of $\{1,2, \ldots, n\}$, we have that $l \leq n$ and by Lemma $3.4, m \leq$ $l$.

Theorem 3.6. The following statements are equivalent:

(i) $X$ is a sublattice of $C(\Omega)$.

(ii) $R(\beta)=\left\{P_{1}, P_{2}, \ldots, P_{n}\right\}$.

Proof. Let $X$ be a sublattice of $C(\Omega)$ and let $\left\{b_{1}, b_{2}, \ldots, b_{n}\right\}$ be a positive basis of $X$. Let $x_{j}=\sum_{i=1}^{n} \lambda_{j i} b_{i}$. Then $z=\sum_{j=1}^{n} x_{j}=\sum_{i=1}^{n} \lambda_{i} b_{i}$ where $\lambda_{i}=\sum_{j=1}^{n} \lambda_{j i}$. Then the sets

$$
I_{i}=b_{i}^{-1}(0,+\infty), \quad i=1,2, \ldots, n,
$$

are pairwise disjoint by Proposition 3.2. Hence for each $t \in I_{k}$ we have $x_{i}(t)=$ $\lambda_{i k} b_{k}(t)$ and $x(t)=\lambda_{k} b_{k}(t)$, and therefore

$$
\beta(t)=\frac{1}{\lambda_{k}}\left(\lambda_{1 k}, \lambda_{2 k}, \ldots, \lambda_{n k}\right)=P_{k} .
$$

Also $D(\beta)=\bigcup_{i=1}^{n} I_{i}$ because $t \in D(\beta)$ iff $z(t)>0$ iff $b_{i}(t)>0$ for at least one $i$. Hence

$$
R(\beta)=\left\{P_{1}, P_{2}, \ldots, P_{n}\right\}
$$

therefore the theorem is true.

Theorem 3.7. Let $Z$ be the sublattice of $C(\Omega)$ generated by $x_{1}, x_{2}, \ldots, x_{n}$ and let $m \in \mathbb{N}$. Then the statements (i) and (ii) are equivalent:

(i) $\operatorname{dim}(Z)=m$.

(ii) $R(\beta)=\left\{P_{1}, P_{2}, \ldots, P_{m}\right\}$.

If the statement (ii) is true, then $Z$ is constructed as follows:

(a) Enumerate $R(\beta)$ so that its $n$ first vectors are linearly independent. (Such an enumeration exists by Lemma 3.4.) Denote again by $P_{i}, i=1,2, \ldots, m$, the new enumeration and let $I_{i}=\beta^{-1}\left(P_{i}\right), i=1,2, \ldots, m$.

(b) Define the functions

$$
x_{n+k}(t)=a_{k}(t)\|r(t)\|_{1}, \quad t \in \Omega, \quad k=1,2, \ldots, m-n,
$$

where $a_{k}$ is the characteristic function of $I_{n+k}$.

(c) $Z=\left[x_{1}, x_{2}, \ldots, x_{n}, x_{n+1}, \ldots, x_{m}\right]$. 
Proof. Suppose that (ii) is true and the assumptions (a), (b) are satisfied. We shall show that (c) is true. It is clear that $m \geq n$. The sets $I_{i}$ are open subsets of $D(\beta)$ because the sets $\left\{P_{i}\right\}$ are open subsets of $R(\beta)$. Also $D(\beta)=\bigcup_{i=1}^{m} I_{i}$. Since $D(\beta)$ is an open subset of $\Omega$, the sets $I_{i}$ are open, nonempty subsets of $\Omega$. Also $\partial\left(I_{i}\right) \cap I_{j}=\emptyset$. Hence $\partial\left(I_{i}\right) \subseteq \Omega \backslash D(\beta)$; therefore $\|r(t)\|_{1}=0$ for each $t \in \partial\left(I_{i}\right)$. This implies that the functions $x_{n+k}$ are continuous; therefore $x_{n+k} \in C_{+}(\Omega)$ for each $k$.

Let $v$ be the curve and $\gamma$ the basic curve of $x_{i}, i=1,2, \ldots, m$. Then by the definition of $x_{n+k}$ we have that

$$
v(t)=(r(t), 0) \quad \text { for each } t \in \bigcup_{i=1}^{n} I_{i}
$$

and

$$
v(t)=\left(r(t),\|r(t)\|_{1} e_{i-n}\right) \quad \text { if } t \in I_{i}, i>n .
$$

Let $t \in I_{i}$. Then

$$
\gamma(t)=(\beta(t), 0)=\left(P_{i}, 0\right)=Q_{i}, \quad \text { if } i \leq n
$$

and

$$
\gamma(t)=\frac{1}{2}\left(\beta(t), e_{i-n}\right)=\frac{1}{2}\left(P_{i}, e_{i-n}\right)=Q_{i}, \quad \text { for each } i=n+1, \ldots, m .
$$

Since $D(\gamma)=D(\beta)=\bigcup_{i=1}^{m} I_{i}$, we have that

$$
R(\gamma)=\left\{Q_{i} \mid i=1,2, \ldots, m\right\}
$$

The vectors $Q_{i}, i=1,2, \ldots, m$, are linearly independent. Hence the functions $x_{i}, i=1,2, \ldots, m$, are also linearly independent; therefore the subspace $Y$ generated by $x_{i}, i=1,2, \ldots, m$, is an $m$-dimensional sublattice of $C(\Omega)$ by the previous theorem. Therefore $Z \subseteq Y$. Since $x_{i}, i=1,2, \ldots, n$, are linearly independent elements of $Z_{+}$and the cardinal number of $R(\beta)$ is $m$, by the statement (iii) of Theorem 3.5 we have that $m \leq \operatorname{dim} Z$. Therefore $\operatorname{dim} Z=m$; hence $Z=Y$.

Suppose now that the statement (i) is true. Then $x_{i}, i=1,2, \ldots, n$, are linearly independent elements of $Z_{+}$; therefore by Theorem 3.5, there exist a nonempty subset $\Phi$ of $\{1,2, \ldots, m\}$ and nonempty, pairwise disjoint open subsets $I_{i}, i \in \Phi$, of $\Omega$ such that $D(\beta)=\bigcup_{i \in \Phi} I_{i}$ and $\beta$ is constant on each $I_{i}$. Hence $R(\beta)=$ $\left\{P_{1}, P_{2}, \ldots, P_{l}\right\}$ where $l$ is the cardinal number of $\Phi$. By the same theorem we have also that $n \leq l \leq m$. As we have proved before, we can construct an $l$-dimensional sublattice $Y$ of $\Omega$ containing $x_{1}, x_{2}, \ldots, x_{n}$; therefore $Z \subseteq Y$ and $m \leq l$. Hence $l=m$ and therefore the statement (ii) is true.

Lattice-subspaces. A subset $K$ of $\mathbb{R}^{l}$ is a polytope if $K$ is the convex hull of a finite subset of $\mathbb{R}^{l}$. The extreme points of $K$ are called vertices of $K$.

Theorem 3.8. Let $Y$ be an l-dimensional lattice-subspace of $C(\Omega)$ containing $x_{1}$, $x_{2}, \ldots, x_{n}$. Suppose that $\left\{b_{1}, b_{2}, \ldots, b_{l}\right\}$ is a positive basis of $Y$,

$$
\begin{aligned}
x_{i} & =\sum_{j=1}^{l} \lambda_{i j} b_{j}, \quad i=1,2, \ldots, n, \\
\sigma_{i} & =\sum_{j=1}^{n} \lambda_{j i}, \quad i=1,2, \ldots, l,
\end{aligned}
$$




$$
\begin{gathered}
\Phi=\left\{i \mid \sigma_{i} \neq 0\right\}, \\
P_{i}=\frac{1}{\sigma_{i}}\left(\lambda_{1 i}, \lambda_{2 i}, \ldots, \lambda_{n i}\right), \quad i \in \Phi,
\end{gathered}
$$

and $K$ is the convex hull of $\overline{R(\beta)}$. Then

(i) $P_{i} \in \overline{R(\beta)}$ for each $i \in \Phi$.

(ii) $K$ is a polytope with vertices $P_{i 1}, P_{i 2}, \ldots, P_{i m}$ where $n \leq m \leq l$ and $i_{\nu} \in \Phi$ for each $\nu=1,2, \ldots, m$.

Proof. Let $x_{n+1}, \ldots, x_{l} \in Y_{+}$such that

$$
Y=\left[x_{1}, x_{2}, \ldots, x_{n}, x_{n+1}, \ldots, x_{l}\right] .
$$

Let

$$
\begin{gathered}
x_{i}=\sum_{j=1}^{l} \lambda_{i j} b_{j}, \quad i=1,2, \ldots, l, \\
s_{i}=\sum_{j=1}^{l} \lambda_{j i}, \quad i=1,2, \ldots, l,
\end{gathered}
$$

and $v(t)=\left(x_{1}(t), x_{2}(t), \ldots, x_{l}(t)\right), t \in \Omega$. Then $\|v(t)\|_{1}=\sum_{i=1}^{l} s_{i} b_{i}$ and the function

$$
\gamma(t)=\frac{v(t)}{\|v(t)\|_{1}}, \quad\|v(t)\|_{1} \neq 0
$$

is the basic curve of $x_{1}, x_{2}, \ldots, x_{l}$. By [12, Proposition 2.3], for each $i=1,2, \ldots, l$ there exists a sequence $\left(\omega_{i \nu}\right)$ of $\Omega$ such that

$$
\lim _{\nu \rightarrow \infty} \frac{b_{j}\left(\omega_{i \nu}\right)}{b_{i}\left(\omega_{i \nu}\right)}=0, \quad \text { for each } j \neq i .
$$

Then

$$
\lim _{\nu \rightarrow \infty} \frac{x_{j}\left(\omega_{i \nu}\right)}{\left\|v\left(\omega_{i \nu}\right)\right\|_{1}}=\lim _{\nu \rightarrow \infty}\left(\frac{\sum_{k=1}^{l} \lambda_{j k} \frac{b_{k}}{b_{i}}}{\sum_{k=1}^{l} s_{k} \frac{b_{k}}{b_{i}}}\right)\left(\omega_{i \nu}\right)=\frac{\lambda_{j i}}{s_{i}}
$$

therefore

$$
\lim _{\nu \rightarrow \infty} \gamma\left(\omega_{i \nu}\right)=\frac{1}{s_{i}}\left(\lambda_{1 i}, \lambda_{2 i}, \ldots, \lambda_{l i}\right)=M_{i} .
$$

Let $A$ be the $l \times l$ matrix with columns the vectors $M_{i}, i=1,2, \ldots, l$. Then using the expansion of $x_{i}$ relative to the positive basis of $Y$ we get

$$
\left(x_{1}, x_{2}, \ldots, x_{l}\right)^{T}=A\left(s_{1} b_{1}, s_{2} b_{2}, \ldots, s_{l} b_{l}\right)^{T} .
$$

Since $\left\{x_{1}, x_{2}, \ldots, x_{l}\right\}$ is also a basis of $Y$, we have that $\operatorname{rank} A=l$; therefore the vectors $M_{i}, i=1,2, \ldots, l$, are linearly independent. Let

$$
\gamma(t)=\sum_{i=1}^{l} \xi_{i}(t) M_{i}
$$

be the expansion of $\gamma(t)$ relative to the basis $\left\{M_{1}, M_{2}, \ldots, M_{l}\right\}$ of $\mathbb{R}^{l}$. Then

$$
(\gamma(t))^{T}=A\left(\xi_{1}(t), \xi_{2}(t), \ldots, \xi_{l}(t)\right)^{T}
$$


and by (4) we get

$$
\left(\xi_{1}(t), \xi_{2}(t), \ldots, \xi_{l}(t)\right)=\frac{1}{\|v(t)\|_{1}}\left(s_{1} b_{1}(t), s_{2} b_{2}(t), \ldots, s_{l} b_{l}(t)\right)
$$

Hence $\xi_{i}(t) \in \mathbb{R}_{+}$and $\sum_{i=1}^{l} \xi_{i}(t)=1$. Therefore $\gamma(t)$ is a convex combination of $M_{1}, M_{2}, \ldots, M_{l}$. Therefore

$$
R(\gamma) \subseteq \operatorname{co}\left\{M_{1}, M_{2}, \ldots, M_{l}\right\}
$$

Let $P(x)=(x(1), x(2), \ldots, x(n)), x \in \mathbb{R}^{l}$, be the natural projection of $\mathbb{R}^{l}$ onto $\mathbb{R}^{n}$. Then

$$
P\left(\frac{s_{i}}{\sigma_{i}} M_{i}\right)=P_{i}, \quad \text { for each } i \in \Phi .
$$

If $i \notin \Phi$, then $P\left(M_{i}\right)=0$, because $\sigma_{i}=0$ and therefore $\lambda_{k i}=0$ for each $k=$ $1,2, \ldots, n$. Also

$$
\beta(t)=\frac{\|v(t)\|_{1}}{\|r(t)\|_{1}} P(\gamma(t)), \quad \text { for each } t \in D(\beta) \subseteq D(\gamma) ;
$$

therefore by (5) we get

$$
\beta(t)=\sum_{i \in \Phi} \frac{\|v(t)\|_{1}}{\|r(t)\|_{1}} \xi_{i}(t) \frac{\sigma_{i}}{s_{i}} P_{i}
$$

Since $\beta(t)$ and $P_{i}$ belong to the simplex $\Delta_{n}$ of $\mathbb{R}_{+}^{n}$, we have that $\beta(t)$ is a convex combination of the vectors $P_{i}, i \in \Phi$; hence

$$
R(\beta) \subseteq \operatorname{co}\left\{P_{i} \mid i \in \Phi\right\}=L .
$$

Since $\Phi$ is finite, the set $L$ is closed; hence $\overline{R(\beta)} \subseteq L$. We shall show that $P_{i} \in \overline{R(\beta)}$, for each $i \in \Phi$. By (3) and (6) we have that $P\left(\frac{s_{i}}{\sigma_{i}} \gamma\left(\omega_{i \nu}\right)\right) \rightarrow P_{i}$. Since $P_{i} \neq 0$, we have that $P\left(\gamma\left(\omega_{i \nu}\right)\right) \neq 0$, for each $\nu$. Therefore $r\left(\omega_{i \nu}\right)=\left\|v\left(\omega_{i \nu}\right)\right\|_{1} P\left(\gamma\left(\omega_{i \nu}\right)\right) \neq 0$; hence $\omega_{i \nu} \in D(\beta)$, for each $\nu$. Similarly with the proof of (3) we can show that $P_{i}=\lim \beta\left(\omega_{i \nu}\right)$. Hence $P_{i} \in \overline{R(\beta)}$; therefore $K=L$. Also $\operatorname{ep}(K) \subseteq\left\{P_{i} \mid i \in \Phi\right\}$. Hence

$$
\operatorname{ep}(K)=\left\{P_{i 1}, P_{i 2}, \ldots, P_{i m}\right\}
$$

where $i_{\nu} \in \Phi$ for $\nu=1,2, \ldots, m$; therefore

$$
K=\operatorname{co}\left\{P_{i 1}, P_{i 2}, \ldots, P_{i m}\right\} .
$$

By Lemma 3.4, the subspace generated by $R(\beta)$, and therefore also by $K$, is the space $\mathbb{R}^{n}$. Hence $\operatorname{ep}(K)$ contains at least $n$ vectors; therefore $n \leq m \leq l$.

Theorem 3.9 ([5, Theorem 2]). Let $d_{1}, d_{2}, \ldots, d_{m} \in \mathbb{R}^{l}$ and let the polytope $D=$ $\operatorname{co}\left\{d_{1}, d_{2}, \ldots, d_{m}\right\}$. Then there exist non-negative, real-valued continuous functions $\xi_{1}, \xi_{2}, \ldots, \xi_{m}$ defined on $D$ such that $x=\sum_{i=1}^{m} \xi_{i}(x) d_{i}$ and $\sum_{i=1}^{m} \xi_{i}(x)=1$, for each $x \in D$.

The previous result in a more general form is given also in [8].

Theorem 3.10. Let the set $K=\operatorname{co} \overline{R(\beta)}$ be a polytope with vertices $P_{1}, P_{2}, \ldots, P_{m}$. Suppose that the $n$ first vertices $P_{1}, P_{2}, \ldots, P_{n}$ of $K$ are linearly independent ${ }^{1}$. Suppose also that $\xi_{i}, i=1,2, \ldots, m$, are positive continuous real-valued functions defined on $D(\beta)$ such that $\sum_{i=1}^{m} \xi_{i}(t)=1$ and $\beta(t)=\sum_{i=1}^{m} \xi_{i}(t) P_{i}$, for each $t \in D(\beta)$.

\footnotetext{
${ }^{1}$ A such enumeration of the vertices of $K$ exists by Lemma 3.4 .
} 
Let $x_{n+i}, i=1,2, \ldots, m-n$, be the functions $x_{n+i}(t)=\xi_{n+i}(t)\|r(t)\|_{1}$ for each $t \in D(\beta)$ and $x_{n+i}(t)=0$ if $t \notin D(\beta)$. Then

$$
Y=\left[x_{1}, x_{2}, \ldots, x_{n}, x_{n+1}, \ldots, x_{m}\right]
$$

is a minimal lattice-subspace of $C(\Omega)$ containing $x_{1}, x_{2}, \ldots, x_{n}$ and $\operatorname{dim} Y=m$.

$A$ positive basis $\left\{b_{1}, b_{2}, \ldots, b_{m}\right\}$ of $Y$ is given by the formula

$$
\left(b_{1}, b_{2}, \ldots, b_{m}\right)^{T}=A^{-1}\left(x_{1}, x_{2}, \ldots, x_{m}\right)^{T}
$$

where $A$ is the $m \times m$ matrix with columns the vectors $R_{i}, i=1,2, \ldots, m$, defined below, in (8).

Proof. We shall show that $Y$ is a lattice-subspace of $C(\Omega)$. Let $v(t)=\left(x_{1}(t), x_{2}(t)\right.$, $\left.\ldots, x_{m}(t)\right), \gamma(t)=\frac{v(t)}{\|v(t)\|_{1}}$ and $l=m-n$. Then

$$
\begin{aligned}
v(t) & =(r(t), 0)+\left(0, \sum_{i=1}^{l} \xi_{n+i}(t)\|r(t)\|_{1} e_{i}\right) \\
& =\|r(t)\|_{1} \sum_{i=1}^{m} \xi_{i}(t)\left(P_{i}, 0\right)+\|r(t)\|_{1} \sum_{i=1}^{l} \xi_{n+i}(t)\left(0, e_{i}\right) \\
& =\|r(t)\|_{1} \sum_{i=1}^{m} \xi_{i}(t) M_{i}, \quad \text { for each } t \in D(\beta)
\end{aligned}
$$

where $M_{i}$ are the following vectors of $\mathbb{R}^{m}$ :

$$
M_{i}=\left(P_{i}, 0\right) \text { for } i=1,2, \ldots, n
$$

and

$$
M_{i}=\left(P_{n+i}, e_{i}\right) \quad \text { for } i=1,2, \ldots, l .
$$

The vectors $M_{i}$ are linearly independent with $\left\|M_{i}\right\|_{1}=1$ for $i=1,2, \ldots, n$ and $\left\|M_{i}\right\|_{1}=2$ for $i=n+1, \ldots, m$. Hence $\|v(t)\|_{1}=\|r(t)\|_{1} g(t)$, where $g(t)=$ $\sum_{i=1}^{m} \xi_{i}(t)\left\|M_{i}\right\|_{1}=1+\sum_{i=n+1}^{m} \xi_{i}(t)$. Therefore, by (7) we have

$$
\gamma(t)=\frac{1}{g(t)} \sum_{i=1}^{m} \xi_{i}(t)\left\|M_{i}\right\|_{1} R_{i}, \quad \text { where } R_{i}=\frac{M_{i}}{\left\|M_{i}\right\|_{1}} .
$$

Hence $\gamma(t)$ is a convex combination of $R_{i}, i=1,2, \ldots, m$. We shall show that $R_{i} \in \overline{R(\gamma)}$ for each $i$. If $P_{i}=\beta\left(t_{i}\right)$, then $P_{i}=\sum_{j=1}^{m} \xi_{j}\left(t_{i}\right) P_{j}$ and by our assumption that $P_{i}$ is an extreme point of $K$, we have that $\xi_{i}\left(t_{i}\right)=1$ and $\xi_{j}\left(t_{i}\right)=0$ for each $j \neq i$. Hence by (8) we have

$$
\gamma\left(t_{i}\right)=\frac{1}{g\left(t_{i}\right)}\left\|M_{i}\right\|_{1} R_{i}=R_{i} .
$$

If $P_{i} \notin R(\beta)$, then there exists a sequence $\left(\omega_{\nu}\right)$ of $D(\beta)$ such that

$$
P_{i}=\lim _{\nu \rightarrow \infty} \beta\left(\omega_{\nu}\right) .
$$

Then

$$
\beta\left(\omega_{\nu}\right)=\sum_{j=1}^{m} \xi_{j}\left(\omega_{\nu}\right) P_{j} .
$$


Since $0 \leq \xi_{j}\left(\omega_{\nu}\right) \leq 1$, there exists a subsequence of $\left(\omega_{\nu}\right)$, which we will denote again by $\left(\omega_{\nu}\right)$ such that

$$
\lambda_{j}=\lim _{\nu \rightarrow \infty} \xi_{j}\left(\omega_{\nu}\right), \quad \text { for each } j=1,2, \ldots, m .
$$

Hence

$$
P_{i}=\sum_{j=1}^{m} \lambda_{j} P_{j},
$$

which implies that $\lambda_{i}=1$ and $\lambda_{j}=0$ for each $j \neq i$, because $P_{i}$ is an extreme point of $K$. By (8) and the definition of $g$ we have that

$$
\lim _{\nu \rightarrow \infty} \gamma\left(\omega_{\nu}\right)=R_{i}
$$

So by Theorem 3.3, $Y$ is a lattice-subspace and a positive basis of $Y$ is as in the formulation of the theorem.

Suppose that $Z \subseteq Y$ is a lattice-subspace containing $x_{1}, x_{2}, \ldots, x_{n}$ and let $\operatorname{dim} Z=l$. Then $l \leq m$. By Theorem 3.8 the number $m$ of vertices of $K$ is less than or equal to $l$; therefore $m=l$. Hence $Z=Y$; therefore $Y$ is minimal.

Definition 3.11. Let $C$ be a convex subset of a normed space $E$. We shall say that $x_{0}$ is a conic point of $C$ if $x_{0}$ is an extreme point of $C, C \backslash\left\{x_{0}\right\} \neq \emptyset$, and there exists a real number $\rho>0$ such that

$$
x_{0}+\rho \frac{x-x_{0}}{\left\|x-x_{0}\right\|} \in C, \quad \text { for each } x \in C, x \neq x_{0} .
$$

Proposition 3.12. Let $D$ be a convex subset of a normed space $E$ and $x_{0} \in E$. If $f^{2} d=d\left(x_{0}, D\right)>0$ and $C=\operatorname{co}\left(\left\{x_{0}\right\} \cup D\right)$, then $x_{0}$ is a conic point of $C$. (If $D$ is bounded and closed, then $C$ is also bounded and closed.)

Proof. Let $x \in C, x \neq x_{0}$. Then $x=\lambda x_{0}+(1-\lambda) y$, where $y \in D$ and $\lambda \in[0,1]$. Hence $x-x_{0}=(1-\lambda)\left(y-x_{0}\right)$; therefore

$$
\left\|x-x_{0}\right\|=(1-\lambda)\left\|y-x_{0}\right\| \geq(1-\lambda) d .
$$

Also $x_{0}+l\left(y-x_{0}\right) \in C$ for each $l \in[0,1]$. Therefore

$$
x_{0}+d \frac{x-x_{0}}{\left\|x-x_{0}\right\|}=x_{0}+\frac{d(1-\lambda)}{\left\|x-x_{0}\right\|}\left(y-x_{0}\right) \in C .
$$

To show that $x_{0}$ is an extreme point of $C$ suppose that $x_{0}=\frac{x_{1}+x_{2}}{2}$ where $x_{1}, x_{2} \in C$ and $x_{1}, x_{2} \neq x_{0}$. Then $x_{i}=\lambda_{i} x_{0}+\left(1-\lambda_{i}\right) y_{i}$ with $\lambda_{i} \in(0,1)$ and $y_{i} \in D$. Then $x_{0}=\frac{1}{2-\lambda_{1}-\lambda_{2}}\left(\left(1-\lambda_{1}\right) y_{1}+\left(1-\lambda_{2}\right) y_{2}\right) \in D$, contradiction. Hence $x_{0}$ is a conic point of $C$.

Example 3.13. (i) For each cone $P \neq\{0\}$ of a normed space, 0 is a conic point of $P$.

(ii) Let $C$ be a closed, convex, bounded subset of a Banach space $E$ and let $x_{0}$ be an extreme point of $C$. If $C=\overline{\operatorname{co}} \operatorname{ep}(C)$ (i.e., $C$ is the closure of the convex hull of the extreme points of $C)$ and $x_{0} \notin D=\overline{\operatorname{co}}\left(\operatorname{ep}(C) \backslash\left\{x_{0}\right\}\right)$, then $C=\operatorname{co}\left(\left\{x_{0}\right\} \cup D\right)$; therefore $x_{0}$ is a conic point of $C$.

(iii) Each vertex of a polytope $C$ of $\mathbb{R}^{m}$ is a conic point of $C$.

\footnotetext{
${ }^{2}$ With $d\left(x_{0}, D\right)$ we denote the distance from $x_{0}$ to $D$.
} 
We prove below that the tangent vector of a curve of $C$ at a conic point of $C$ is equal to zero.

Proposition 3.14. Let $C$ be a closed, convex subset of a normed space $E$ and $x_{0}$ be a conic point of $C$. Let $\phi:(-\epsilon, \epsilon) \rightarrow C$ be a function with $\phi(0)=x_{0}$ where $\epsilon$ is a positive real number. Then

$$
\phi^{\prime}(0)=0
$$

whenever the derivative $\phi^{\prime}(0)$ exists.

Proof. Let $\phi^{\prime}(0)=\lim _{t \rightarrow 0} \frac{\phi(t)-\phi(0)}{t} \neq 0$. Then there exists $\delta>0$ such that $\phi(t) \neq$ $\phi(0)$ for each $|t|<\delta$. Hence

$$
\lim _{t \rightarrow 0_{+}} \frac{\phi(t)-\phi(0)}{\|\phi(t)-\phi(0)\|}=\lim _{t \rightarrow 0_{+}} \frac{\phi(t)-\phi(0)}{t} \cdot \lim _{t \rightarrow 0_{+}} \frac{1}{\left\|\frac{\phi(t)-\phi(0)}{t}\right\|}=\frac{\phi^{\prime}(0)}{\left\|\phi^{\prime}(0)\right\|},
$$

and similarly

$$
\lim _{t \rightarrow 0_{-}} \frac{\phi(t)-\phi(0)}{\|\phi(t)-\phi(0)\|}=-\frac{\phi^{\prime}(0)}{\left\|\phi^{\prime}(0)\right\|} .
$$

Since $x_{0}$ is a conic point of $C$, there exists $\rho>0$ such that

$$
x_{0}+\rho \frac{x-x_{0}}{\left\|x-x_{0}\right\|} \in C, \quad \text { for each } x \in C, x \neq x_{0} .
$$

Therefore

$$
\lim _{\nu \rightarrow \infty}\left(\phi(0)+\rho \frac{\phi(1 / \nu)-\phi(0)}{\|\phi(1 / \nu)-\phi(0)\|}\right)=x_{0}+\rho \frac{\phi^{\prime}(0)}{\left\|\phi^{\prime}(0)\right\|}=z_{1} \in C
$$

and

$$
\lim _{\nu \rightarrow \infty}\left(\phi(0)+\rho \frac{\phi(-1 / \nu)-\phi(0)}{\|\phi(-1 / \nu)-\phi(0)\|}\right)=x_{0}-\rho \frac{\phi^{\prime}(0)}{\left\|\phi^{\prime}(0)\right\|}=z_{2} \in C .
$$

Hence $x_{0}=\frac{1}{2}\left(z_{1}+z_{2}\right)$, contradiction. Therefore $\phi^{\prime}(0)=0$.

Corollary 3.15. Let the set $K=\operatorname{co} \overline{R(\beta)}$ be a polytope of $\mathbb{R}^{n}$ and let $\beta\left(t_{0}\right)$ be a vertex of $K$. If $\epsilon$ is a positive real number and $g:(-\epsilon, \epsilon) \rightarrow \Omega$ is a function with $g(0)=t_{0}$ and $\phi(\lambda)=\beta(g(\lambda))$, then

$$
\phi^{\prime}(0)=0
$$

whenever the derivative exists.

Remark 3.16. Suppose that there exists a finite-dimensional lattice-subspace of $C(\Omega)$ containing $X$. Then $K$ is a polytope of $\mathbb{R}^{n}$. Suppose that $\beta\left(t_{0}\right)$ is a vertex of $K$. If $c$ is a curve of $\Omega$ and $t_{0}$ an interior point of $c$, then the derivative at $t_{0}$ of the restriction of $\beta$ on the curve $c$ is equal to zero.

If for example $\Omega \subseteq \mathbb{R}^{l}$, then the partial derivatives of $\beta$ at $t_{0}$ are equal to zero whenever $t_{0} \in \operatorname{int}(\Omega)$. If $t_{0} \in \partial(\Omega)$, the derivatives at $t_{0}$ of the restriction of $\beta$ on the parametrics curves of $\partial(\Omega)$ are equal to zero.

Algorithm 3.17. Theorem 3.10 and Corollary 3.15 define a process which in many cases, especially when $\Omega \subseteq \mathbb{R}^{l}$, determines whether a finite dimensional minimal lattice-subspace exists and determines also a positive basis of these subspaces. To study this problem we study if $K$ is a polytope or not. 
If the set $R(\beta)$ is closed, then each extreme point (vertex) $P_{0}$ of $K=\operatorname{co} R(\beta)$ belongs to $R(\beta)$; therefore $P_{0}=\beta\left(t_{0}\right)$. Also the geometry of the boundary of $D(\beta)$ and the differentiability of the functions $x_{i}$ are very important for this study.

Let $\Omega=[a, b]$, the functions $x_{i}$ are differentiable and $D(\beta)=\Omega$. Suppose that the set $K$ is a polytope with vertices $\beta\left(t_{i}\right), i=1,2, \ldots, m$. Then at least $m-2$ of $t_{i}$ belong to $(a, b)$; therefore the equation

$$
\beta^{\prime}(t)=0,
$$

where $\beta^{\prime}$ is the derivative of $\beta$, has at least $m-2$ roots in $(a, b)$. Hence the vertices of $K$ belong to the set

$$
G=\{\beta(t) \mid t=a, t=b, \text { or } t \text { is a root of }(9)\}
$$

which we call the set of possible vertices of $K$. Let $D=\operatorname{co} G$. It is easy to show that $K$ is a polytope if and only if $D$ is a polytope and $R(\beta) \subseteq D$.

Hence in this case the algorithm is the following:

(i) Determine equation (9). If this equation does not have at least $n-2$ roots in $(a, b)$, then $K$ is not a polytope.

(ii) Determine the roots $t_{i}$ of $(9)$ in $(a, b)$.

(iii) We study whether $R(\beta) \subseteq D$. So we study whether $\beta(t)$ is a convex combination of $\beta(a), \beta(b), \beta\left(t_{i}\right)$, for each $i$. If $R(\beta) \nsubseteq D$, then $K$ is not a polytope.

(iv) Determine the vertices of $K$ and a positive basis of the minimal latticesubspace, in accordance with Theorem 3.10.

We give three examples below. In (i) it is shown that a finite-dimensional minimal lattice-subspace does not always exist. In (ii) we consider three elements $x_{1}, x_{2}, x_{3}$ of $C(\Omega)$, where $\Omega$ is a square of $\mathbb{R}^{2}$. We show that a 4 -dimensional minimal latticesubspace $Y$ exists and a positive basis of $Y$ is determined. We also remark that the sublattice generated by the elements $x_{i}$ is dense in $C(\Omega)$. In (iii) the functions $x_{i}$ are as in (ii), but $\Omega$ is a circle of $\mathbb{R}^{2}$. It is shown that a finite-dimensional minimal lattice-subspace does not exist. This difference between (ii) and (iii) depends on the geometry of the boundary of $\Omega$.

Example 3.18. (i) Let $\Omega=[0,1], x_{1}(t)=1, x_{2}(t)=t, x_{3}(t)=t^{2}$. Then

$$
\beta(t)=\left(\frac{1}{1+t+t^{2}}, \frac{t}{1+t+t^{2}}, \frac{t^{2}}{1+t+t^{2}}\right), \quad t \in[0,1],
$$

is the basic curve of $x_{1}, x_{2}, x_{3}$ and $\beta^{\prime}(t) \neq 0$ for each $t \in(0,1)$. Suppose that $Y$ is a finite-dimensional lattice-subspace of $C(\Omega)$ containing the functions $x_{i}$. Then $\operatorname{dim} Y \geq 3$, and therefore by Theorem $3.8 \mathrm{~K}$ is a polytope of $\mathbb{R}^{3}$ with at least three vertices, $\beta\left(t_{1}\right), \beta\left(t_{2}\right), \beta\left(t_{3}\right)$. Hence $\beta^{\prime}(t)=0$ for at least one point of $(0,1)$, contradiction.

(ii) Let $\Omega=[0,1] \times[0,1], x_{1}(u, v)=1, x_{2}(u, v)=u, x_{3}(u, v)=v$ and $X=$ $\left[x_{1}, x_{2}, x_{3}\right]$. Then

$$
\beta(u, v)=\left(\frac{1}{1+u+v}, \frac{u}{1+u+v}, \frac{v}{1+u+v}\right), \quad(u, v) \in \Omega,
$$

is the basic curve of $x_{1}, x_{2}, x_{3}$ and let $K=\operatorname{co} R(\beta)$. Since the range of $\beta$ is not finite, the sublattice $Z$ generated by $x_{1}, x_{2}, x_{3}$ is an infinite-dimensional subspace of $C(\Omega)$, Theorem 3.7. In this example we can also show that $Z$ is dense in $C(\Omega)$ because $Z$ is a sublattice of $C(\Omega)$ and $Z$ contains the constant functions. 

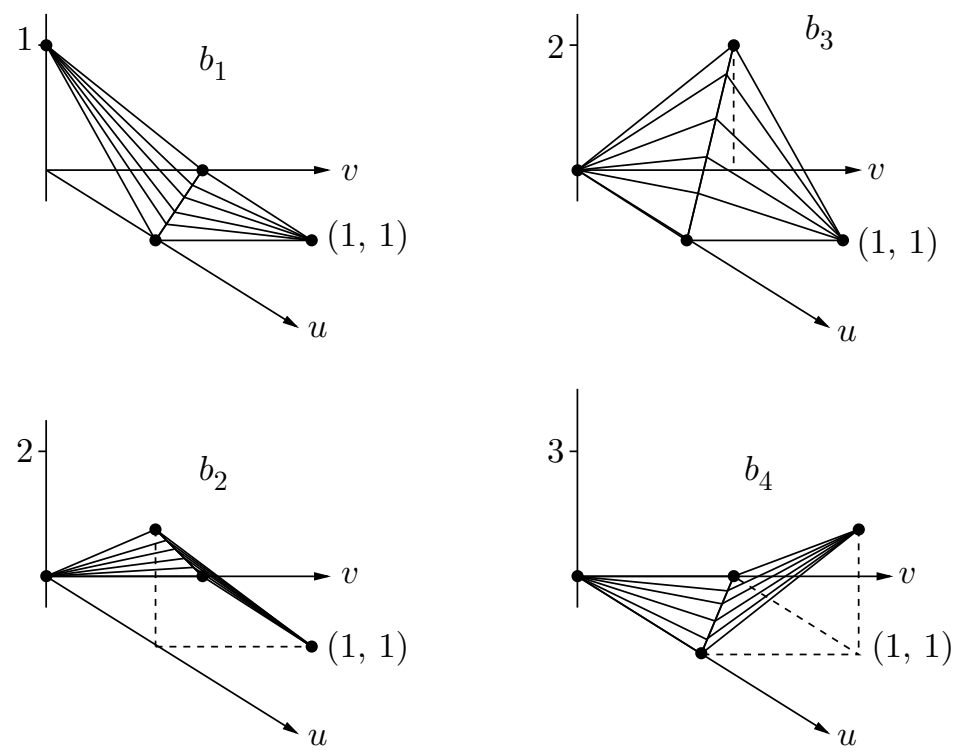

FIGURE 1

In order to study the existence of minimal lattice-subspaces we study whether the set $K$ is a polytope of $\mathbb{R}^{3}$. To this end suppose that $K$ is a polytope. Then by Theorem 3.8, $K$ has at least three vertices and let $\beta\left(t_{0}\right)$ be a vertex of $K$. Then $t_{0}$ is also a vertex of $\Omega$ because in the contrary case $t_{0}$ will be an interior point of a line segment parallel to an axis of $\mathbb{R}^{2}$; therefore, and by the previous corollary, at least one of the partial derivatives of $\beta$ at $t_{0}$ will be equal to zero, contradiction. Hence the points $P_{1}=\beta(0,0)=(1,0,0), P_{2}=\beta(1,0)=(1 / 2,1 / 2,0), P_{3}=\beta(0,1)=$ $(1 / 2,0,1 / 2)$ and $P_{4}=\beta(1,1)=(1 / 3,1 / 3,1 / 3)$ define the set of possible vertices of $K$. Let $D=\operatorname{co}\left\{P_{1}, P_{2}, P_{3}, P_{4}\right\}$. From the above remarks we have that $K$ is a polytope if and only if $K=D$ or equivalently if $R(\beta) \subseteq D$. It is easy to show that

$$
\beta(u, v)=\sum_{i=1}^{4} \xi_{i}(u, v) P_{i},
$$

where $\xi_{1} \in C(\Omega), \xi_{2}(u, v)=2\left(\frac{1-v}{1+u+v}-\xi_{1}(u, v)\right), \xi_{3}(u, v)=2\left(\frac{1-u}{1+u+v}-\xi_{1}(u, v)\right)$ and $\xi_{4}(u, v)=3\left(\frac{u+v-1}{1+u+v}+\xi_{1}(u, v)\right)$.

Since $\beta(u, v)$ and the points $P_{i}$ belong to the plane $x(1)+x(2)+x(3)=1$ of $\mathbb{R}^{3}$ we have that $\sum_{i=1}^{4} \xi_{i}(u, v)=1$. If $\xi(u, v)=\frac{1-u-v}{1+u+v}$ and if we put $\xi_{1}=\xi^{+}$, then the functions $\xi_{i}, i=1,2,3,4$, are positive and continuous; therefore $R(\beta) \subseteq D$. Hence $K$ is a polytope with vertices $P_{i}, i=1,2,3,4$, and the three first of them are linearly independent. By Theorem 3.10,

$$
Y=\left[x_{1}, x_{2}, x_{3}, x_{4}\right]
$$

where $x_{4}(u, v)=\xi_{4}(u, v)\|r(u, v)\|_{1}=3(1-u-v)^{+}$, is a minimal lattice-subspace containing $x_{1}, x_{2}, x_{3}$.

A positive basis $\left\{b_{1}, b_{2}, b_{3}, b_{4}\right\}$ of $Y$ is given by the formula

$$
\left(b_{1}, b_{2}, b_{3}, b_{4}\right)^{T}=A^{-1}\left(x_{1}, x_{2}, x_{3}, x_{4}\right)^{T},
$$


where $A$ is the $4 \times 4$ matrix with columns the vectors $R_{i}=\frac{M_{i}}{\left\|M_{i}\right\|_{1}}, i=1,2,3,4$, and $M_{1}=\left(P_{1}, 0\right)=(1,0,0,0), M_{2}=\left(P_{2}, 0\right)=(1 / 2,1 / 2,0,0), M_{3}=\left(P_{3}, 0\right)=$ $(1 / 2,0,1 / 2,0), M_{4}=\left(P_{4}, e_{1}\right)=(1 / 3,1 / 3,1 / 3,1)$.

After the computations we get

$$
\begin{aligned}
& b_{1}(u, v)=x_{1}-x_{2}-x_{3}+\frac{1}{3} x_{4}= \begin{cases}1-u-v & \mid u+v \leq 1, \\
0 & \mid u+v>1,\end{cases} \\
& b_{2}(u, v)=2 x_{2}-\frac{2}{3} x_{4}= \begin{cases}2 u & \mid u+v \leq 1, \\
2(1-v) & \mid u+v>1,\end{cases} \\
& b_{3}(u, v)=2 x_{3}-\frac{2}{3} x_{4}= \begin{cases}2 v & \mid u+v \leq 1, \\
2(1-u) & \mid u+v>1,\end{cases} \\
& b_{4}(u, v)=2 x_{4}=\left\{\begin{array}{ll}
0 & \mid u+v \leq 1, \\
3(u+v-1) & \mid u+v>1
\end{array}\right. \text { (Figure 1). }
\end{aligned}
$$

(iii) Let $\Omega=\left\{(u, v) \in \mathbb{R}^{2} \mid u^{2}+v^{2} \leq 1\right\}$ and let $x_{i}, i=1,2,3$, be the functions of the previous example. Suppose that $K$ is a polytope and $\beta\left(t_{0}\right)$ a vertex of $K$. As before we have that $t_{0} \in \partial(\Omega)$ and let $t_{0}=\left(\cos \theta_{0}, \sin \theta_{0}\right)$. Then by the corollary we have $\phi^{\prime}\left(\theta_{0}\right)=0$ where $\phi(\theta)=\beta(\cos \theta, \sin \theta)$. This is a contradiction because $\phi^{\prime}(\theta) \neq 0$ for each $\theta$. Therefore a finite-dimensional lattice-subspace containing the functions $x_{i}$ does not exist.

To study subspaces of $\mathbb{R}^{l}, l>1$, suppose that $\Omega=\{1,2, \ldots, l\}$. Then $C(\Omega)=\mathbb{R}^{l}$,

$$
x_{i}=\left(x_{i}(1), x_{i}(2), \ldots, x_{i}(l)\right), \quad i=1,2, \ldots, n,
$$

are linearly independent, positive elements of $\mathbb{R}^{l}$ and

$$
X=\left[x_{1}, x_{2}, \ldots, x_{n}\right] .
$$

The curve $r$ and the basic curve $\beta$ of the vectors $x_{i}, i=1,2, \ldots, n$, are the functions:

$$
r(i)=\left(x_{1}(i), x_{2}(i), \ldots, x_{n}(i)\right), \quad i=1,2, \ldots, l,
$$

and

$$
\beta(i)=\frac{r(i)}{\|r(i)\|_{1}}, \quad \text { for each } i \text { with }\|r(i)\|_{1} \neq 0 .
$$

Let $m$ be the cardinal number of $R(\beta)$. Then $m \leq l$ and by Lemma 3.4, $n \leq m$; therefore $n \leq m \leq l$. Let $K$ be the convex hull of $R(\beta)$. Then $K$, as the convex hull of a finite subset of $\mathbb{R}^{n}$, is a polytope with $d$ vertices. It is clear that

$$
n \leq d \leq m \leq l
$$

and that each vertex of $K$ belongs to $R(\beta)$. Let

$$
R(\beta)=\left\{P_{1}, P_{2}, \ldots, P_{m}\right\}
$$

be an enumeration of $R(\beta)$ such that:

(i) the vectors $P_{i}, i=1,2, \ldots, n$, are linearly independent and

(ii) the points $P_{i}, i=1,2, \ldots, d$, are the vertices of $K$.

As an application of Theorems 3.6, 3.3, 3.7 and 3.10 we obtain the following:

Theorem 3.19 (The case of $\mathbb{R}^{l}$ ). Suppose that $\Omega=\{1,2, \ldots, l\}$ and that the above assumptions are satisfied. Then 
(i) $X$ is a sublattice of $\mathbb{R}^{l}$ if and only if $R(\beta)$ contains exactly $n$ points (i.e., $m=n)$.

(ii) $X$ is a lattice-subspace of $\mathbb{R}^{l}$ if and only if the polytope $K$ has $n$ vertices (i.e., $d=n)$.

(iii) Let $m>n$. If $I_{k}=\beta^{-1}\left(P_{k}\right)$, and

$$
x_{k}=\sum_{i \in I_{k}}\|r(i)\|_{1} e_{i}, \quad k=n+1, n+2, \ldots, m,
$$

then

$$
Z=\left[x_{1}, \ldots, x_{n}, x_{n+1}, \ldots, x_{m}\right]
$$

is the sublattice generated by $x_{1}, x_{2}, \ldots, x_{n}$ and $\operatorname{dim} Z=m$.

(iv) Let $d>n$. If $\xi_{i}: D(\beta) \rightarrow \mathbb{R}_{+}, i=1,2, \ldots, d$, such that $\sum_{i=1}^{d} \xi_{i}(j)=1$ and $\beta(j)=\sum_{i=1}^{d} \xi_{i}(j) P_{i}$ for each $j \in D(\beta)$, and $x_{n+i}, i=1,2, \ldots, d-n$, are the following vectors of $\mathbb{R}^{l}$ :

$$
x_{n+i}=\sum_{j \in D(\beta)} \xi_{n+i}(j)\|r(j)\|_{1} e_{j},
$$

then

$$
Y=\left[x_{1}, \ldots, x_{n}, x_{n+1}, \ldots, x_{d}\right]
$$

is a minimal lattice-subspace of $\mathbb{R}^{l}$ containing $x_{1}, x_{2}, \ldots, x_{n}$ and $\operatorname{dim} Y=d$.

In the following result $\Omega$ is again a compact, Hausdorff, topological space.

Theorem 3.20. Let $K=\operatorname{co} \overline{R(\beta)}$ and let $L$ be the set of finite-dimensional minimal lattice-subspaces of $C(\Omega)$ containing $x_{1}, x_{2}, \ldots, x_{n}$. Then the following statements are equivalent:

(i) $K$ is a polytope with $m$ vertices.

(ii) $L \neq \emptyset$ and $\operatorname{dim} Y=m$, for each $Y \in L$.

(iii) $L \neq \emptyset$.

Proof. Suppose that (i) is true. Then by Theorem 3.10, there exists $Y \in L$ with $\operatorname{dim} Y=m$. Suppose that $Z \in L$ and $\left\{b_{1}, b_{2}, \ldots, b_{l}\right\}$ is a positive basis of $Z$. Let

$$
\begin{gathered}
x_{i}=\sum_{j=1}^{l} \lambda_{i j} b_{j}, \quad i=1,2, \ldots, n, \\
\sigma_{j}=\sum_{i=1}^{n} \lambda_{i j}, \quad j=1,2, \ldots, l, \\
\Phi=\left\{j \mid \sigma_{j} \neq 0\right\} \quad \text { and } \\
P_{i}=\frac{1}{\sigma_{i}}\left(\lambda_{1 i}, \lambda_{2 i}, \ldots, \lambda_{n i}\right), \quad i \in \Phi .
\end{gathered}
$$

Then by Theorem $3.8 P_{i} \in K$ for each $i \in \Phi$ and the vertices of $K$ are among the points $P_{i}, i \in \Phi$; therefore there exist $i_{1}, i_{2}, \ldots, i_{m} \in \Phi$ such that $P_{i 1}, P_{i 2}, \ldots, P_{i m}$ 
are the vertices of $K$. Also $n \leq m \leq l$. Let $T: Z \rightarrow \mathbb{R}^{l}$ such that $T\left(\sum_{i=1}^{l} \xi_{i} b_{i}\right)=$ $\sum_{i=1}^{l} \xi_{i} e_{i}$ and let $y_{i}=T\left(x_{i}\right), i=1,2, \ldots, n$. The basic curve $b$ of $y_{1}, y_{2}, \ldots, y_{n}$ is:

$$
b(i)=\frac{1}{\sigma_{i}}\left(\lambda_{1 i}, \lambda_{2 i}, \ldots, \lambda_{n i}\right), \quad i \in \Phi,
$$

with range

$$
R(b)=\left\{P_{i} \mid i \in \Phi\right\} .
$$

So $R(b)$ is a subset of $K$ containing the vertices of $K$; therefore

$$
K=\operatorname{co} R(b) \text {. }
$$

Hence co $R(b)$ is a polytope with vertices $P_{i 1}, P_{i 2}, \ldots, P_{i m}$. By the previous theorem, there exists an $m$-dimensional lattice-subspace $F$ of $\mathbb{R}^{l}$ containing $y_{1}, y_{2}, \ldots$, $y_{n}$. If $G=T^{-1}(F)$, then $G$ is a lattice-subspace of $Z$ and therefore also of $C(\Omega)$ containing $x_{1}, x_{2}, \ldots, x_{n}$. Since $Z$ is minimal, we have that $G=Z$, and therefore $\operatorname{dim} Z=\operatorname{dim} F=m$. Hence we have shown that (i) $\Rightarrow$ (ii).

Suppose now that the statement (ii) is true. Let $Y \in L$ and $K=\operatorname{co} \overline{R(\beta)}$. Then by Theorem 3.8, $K$ is a polytope with $k$ vertices and

$$
n \leq k \leq m .
$$

By Theorem 3.10 there exists $Z \in L$ with $\operatorname{dim} Z=k$. By our assumption we have that $k=m$; therefore $K$ has $m$ vertices. Hence (ii) $\Rightarrow$ (i).

Also (ii) $\Rightarrow$ (iii) and (iii) $\Rightarrow$ (i) by Theorem 3.8.

In the following example we construct the sublattice $Z$ generated by a fourdimensional subspace $X$ of $\mathbb{R}^{7}$ as well as two minimal lattice-subspaces $Y$ and $Y^{\prime}$ which contain $X$. It is remarkable that $Y \cap Y^{\prime}$ is not a lattice-subspace as well as that both $Y$ and $Y^{\prime}$ are not subspaces of $Z$.

Example 3.21. Let

$$
\begin{aligned}
& x_{1}=(1,2,1,0,1,1,4), \\
& x_{2}=(0,1,1,1,1,0,2), \\
& x_{3}=(2,1,0,1,1,1,2), \\
& x_{4}=(1,0,1,1,1,0,0),
\end{aligned}
$$

and let $X=\left[x_{1}, x_{2}, x_{3}, x_{4}\right]$. Let $r$ be the curve and $\beta$ the basic curve of $x_{i}, i=$ $1,2,3,4$. Then $r(1)=(1,0,2,1), r(2)=(2,1,1,0), r(3)=(1,1,0,1), r(4)=$ $(0,1,1,1), r(5)=(1,1,1,1), r(6)=(1,0,1,0), r(7)=(4,2,2,0)$ and $\beta(1)=$ $\frac{1}{4}(1,0,2,1), \beta(2)=\beta(7)=\frac{1}{4}(2,1,1,0), \beta(3)=\frac{1}{3}(1,1,0,1), \beta(4)=\frac{1}{3}(0,1,1,1)$, $\beta(5)=\frac{1}{4}(1,1,1,1), \beta(6)=\frac{1}{2}(1,0,1,0)$. In order to enumerate $R(\beta)$ as in Theorem 3.19 we remark the following:

(i) The vectors $P_{1}=\beta(4), P_{2}=\beta(1), P_{3}=\beta(6)$ and $P_{4}=\beta(3)$ are linearly independent.

(ii) Let $\beta(2)=P_{5}$. Then it is easy to show that for any proper subset $\Phi$ of $\left\{P_{1}, P_{2}, P_{3}, P_{4}, P_{5}\right\}, \operatorname{co} \Phi \neq \operatorname{co}\left\{P_{1}, P_{2}, P_{3}, P_{4}, P_{5}\right\}=K$; therefore $P_{i}, \quad i=$ $1,2,3,4,5$, are vertices of the polytope $K$.

(iii) It is easy also to show that

$$
\beta(5)=\frac{3(1-\theta)}{8} P_{1}+\theta P_{2}+\frac{1-5 \theta}{4} P_{3}+\frac{3(1-\theta)}{8} P_{4}+\theta P_{5}
$$


Hence for any $\theta \in\left[0, \frac{1}{5}\right]$ the vector $P_{6}=\beta(5)$ is a convex combination of $P_{i}, i=1,2,3,4,5$; therefore $P_{6} \in K$.

Hence

$$
R(\beta)=\left\{P_{1}, P_{2}, P_{3}, P_{4}, P_{5}, P_{6}\right\}
$$

and in accordance with the notations of Theorem 3.19, $n=4, d=5$ and $m=6$. Since $n<d, X$ is not a lattice-subspace and therefore also $X$ is not a sublattice of $\mathbb{R}^{7}$. Let $Z$ be the sublattice of $\mathbb{R}^{7}$ generated by $x_{1}, x_{2}, x_{3}, x_{4}$. In order to determine $Z$ we define the sets

$$
I_{5}=\beta^{-1}\left(P_{5}\right)=\{2,7\}, \quad I_{6}=\beta^{-1}\left(P_{6}\right)=\{5\}
$$

and the vectors

$$
x_{5}=\|r(2)\|_{1} e_{2}+\|r(7)\|_{1} e_{7}=4 e_{2}+8 e_{7}
$$

and

$$
x_{6}=\|r(5)\|_{1} e_{5}=4 e_{5} .
$$

Then by the theorem

$$
Z=\left[x_{1}, x_{2}, x_{3}, x_{4}, x_{5}, x_{6}\right] .
$$

By Theorem 3.3 a positive basis $\left\{b_{1}, b_{2}, b_{3}, b_{4}, b_{5}, b_{6}\right\}$ of $Z$ is given by the formula

$$
\left(b_{1}, b_{2}, b_{3}, b_{4}, b_{5}, b_{6}\right)^{T}=A^{-1}\left(x_{1}, x_{2}, x_{3}, x_{4}, x_{5}, x_{6}\right)^{T},
$$

where $A$ is the $6 \times 6$ matrix with columns the vectors $\gamma(i), i=1,2, \ldots, 6$, and $\gamma$ is the basic curve of the vectors $x_{i}, i=1,2, \ldots, 6$. So after the computations we find that $b_{1}=4 e_{1}, b_{2}=8 e_{2}+16 e_{7}, b_{3}=3 e_{3}, b_{4}=3 e_{4}, b_{5}=8 e_{5}$ and $b_{6}=2 e_{6}$.

To determine a minimal lattice-subspace define the vectors $\xi_{i}, i=1,2,3,4,5$, of $\mathbb{R}^{7}$ such that

$$
\begin{aligned}
& \sum_{i=1}^{5} \xi_{i}(j)=1 \quad \text { and } \quad \beta(j)=\sum_{i=1}^{5} \xi_{i}(j) P_{i}, \quad \text { for each } j=1,2, \ldots, 7 . \\
& \beta(1)=P_{2}=\sum_{i=1}^{5} \xi_{i}(1) P_{i} \quad \Rightarrow \quad \xi_{2}(1)=1 \text { and } \xi_{k}(1)=0 \text { for } k \neq 2 . \\
& \beta(2)=P_{5}=\sum_{i=1}^{5} \xi_{i}(2) P_{i} \quad \Rightarrow \quad \xi_{5}(2)=1 \text { and } \xi_{k}(2)=0 \text { for } k \neq 5 \text {. } \\
& \beta(3)=P_{4}=\sum_{i=1}^{5} \xi_{i}(3) P_{i} \quad \Rightarrow \quad \xi_{4}(3)=1 \text { and } \xi_{k}(3)=0 \text { for } k \neq 4 \text {. } \\
& \beta(4)=P_{1}=\sum_{i=1}^{5} \xi_{i}(4) P_{i} \quad \Rightarrow \quad \xi_{1}(4)=1 \text { and } \xi_{k}(4)=0 \text { for } k \neq 1 \text {. } \\
& \beta(5)=P_{6}=\sum_{i=1}^{5} \xi_{i}(5) P_{i} \Rightarrow \xi_{1}(5)=\xi_{4}(5)=\frac{3(1-\theta)}{8}, \xi_{2}(5)=\xi_{5}(5)=\theta \text {, } \\
& \xi_{3}(5)=\frac{1-5 \theta}{4} \text {, by (10). } \\
& \beta(6)=P_{3}=\sum_{i=1}^{5} \xi_{i}(6) P_{i} \quad \Rightarrow \quad \xi_{3}(6)=1 \text { and } \xi_{k}(6)=0 \text { for } k \neq 3 \text {. } \\
& \beta(7)=P_{2}=\sum_{i=1}^{5} \xi_{i}(7) P_{i} \quad \Rightarrow \quad \xi_{2}(7)=1 \text { and } \xi_{k}(7)=0 \text { for } k \neq 2 \text {. }
\end{aligned}
$$

Define also the vector

$$
\begin{aligned}
y_{5} & =\sum_{j=1}^{7} \xi_{5}(j)\|r(j)\|_{1} e_{j}=\|r(2)\|_{1} e_{2}+\theta\|r(5)\|_{1} e_{5} \\
& =4 e_{2}+4 \theta e_{5}, \quad \theta \in[0,1 / 5] .
\end{aligned}
$$


Suppose that $\theta>0$ in $y_{5}$ and that $y_{5}^{\prime}$ is the vector corresponding to $\theta=0$, i.e., $y_{5}^{\prime}=4 e_{2}$. Then the subspaces

$$
Y=\left[x_{1}, x_{2}, x_{3}, x_{4}, y_{5}\right] \quad \text { and } \quad Y^{\prime}=\left[x_{1}, x_{2}, x_{3}, x_{4}, y_{5}^{\prime}\right]
$$

are minimal lattice-subspaces containing the vectors $x_{i}$. Since the vectors $x_{1}, x_{2}, x_{3}$, $x_{4}, y_{5}, y_{5}^{\prime}$ are linearly independent, we have $Y \neq Y^{\prime}$. Also $X=Y \cap Y^{\prime}$ is not a latticesubspace. An important remark is that the vectors $y_{5}, y_{5}^{\prime}$ do not belong to $Z$. To show this suppose that $y_{5} \in Z$. Then $y_{5} \in Z_{+}$, and therefore

$$
y_{5}=\sum_{i=1}^{6} \lambda_{i} b_{i}, \quad \text { with } \lambda_{i} \in \mathbb{R}_{+} \text {for each } i .
$$

This implies that $\lambda_{2}=1 / 2$ and $\lambda_{2}=0$, contradiction. Hence $y_{5} \notin Z$. Also $y_{5}^{\prime} \notin Z$. Therefore $Y, Y^{\prime}$ are not subspaces of $Z$.

\section{REFERENCES}

1. Y. A. Abramovich, C. D. Aliprantis, and I. A. Polyrakis, Lattice-subspaces and positive projections, Proc. Roy. Irish Acad. 94 A (1994), no. 2, 237-253. MR 87b:46010

2. C. D. Aliprantis, D. Brown, I. Polyrakis, and J. Werner, Portfolio dominance and optimality in infinite security markets, J. Math. Economics 30 (1998), 347-366. CMP 99:04

3. C. D. Aliprantis, D. Brown, and J. Werner, Minimum-cost portfolio insurance, J. Economic Dynamics and Control (to appear).

4. C. D. Aliprantis and O. Burkinshaw, Positive operators, Academic Press, New York \& London, 1985. MR 87h: 47086

5. J. A. Kalman, Continuity and convexity of projections and barycentric coordinates in convex polyhedra, Pacific J. of Math. (1961), 1017-1022. MR 24:A3557

6. P. Meyer-Nieberg, Banach lattices, Springer-Verlag, 1991. MR 93f:46025

7. S. Miyajima, Structure of Banach quasi-sublattices, Hokkaido Math. J. 12 (1983), 83-91. MR 84g:46033

8. S. Papadopoulou, On the geometry of stable compact convex sets, Math. Annalen 229 (1977), 193-200. MR 56:9228

9. A. Peresini, Ordered topological vector spaces, Harper \& Row, New York, 1967.

10. I. A. Polyrakis, Schauder bases in locally solid lattice Banach spaces, Math. Proc. Cambridge Philos. Soc. 101 (1987), 91-105. MR 89b:46020

11. L Lattice-subspaces of $C[0,1]$ and positive bases, J. Math. Anal. Appl. 184 (1994), 1-18. MR 95g:46040

12. - Finite-dimentional lattice-subspaces of $C(\Omega)$ and curves of $\mathbb{R}^{n}$, Trans. American Math. Soc. 384 (1996), 2793-2810. MR 96k:46031

Department of Mathematics, National Technical University of Athens, Zographou 157 80, Athens, Greece

E-mail address: ypoly@math.ntua.gr 